\title{
ECUACIÓN VISCOELĀSTICA CON AMORTIGUAMIENTO LOCALMENTE DISTRIBUIDO
}

\author{
Alfonso Pérez Salvatierra ${ }^{1}$, Victoriano Yauri Luque ${ }^{2}$, \\ Andrés Guardia Cayo ${ }^{3}$, Elvis Bustamante ${ }^{4}$
}

\begin{abstract}
Resumen: En el presente trabajo estudiamos la ecuación viscoelastica unidimensional definida sobre el intervalo $[0, L]$. Dividimos el estudio en dos partes:

En la primera analizamos la existencia y unicidad de soluciones usando la teoría de los semigrupos lineales, aplicando en el problema de Cauchy Abstracto y en la segunda parte vemos la estabilidad exponencial del $C_{0}$ - semigrupo de contracciones asociado al sist.ma viscoelástica lineal.
\end{abstract}

Palabras clave: Ecuación viscoelástica, semigrupo, generador infinitesimal .

\section{VISCOELASTIC EQUATION WITH LOCALLY DISTRIBUTED DAMPING}

Abstract: In this present work we study the one-dimensional viscoelastic equation defined on the interval $[0, L]$. We divided the study into two parts:

In the first we analyze the existence and uniqueness of solutions using the theory of the linear semigroups, applying in the Abstract Cauchy problem and in the second part we see the exponential stability of the $C_{0}$-semigroup of contractions associated with linear viscoelastic system.

Key words: Viscoelastic equation, semigroup, infinitesimal generator.

\section{Introducción}

Consideraremos el movimiento de una barra elástica, $u(x, t)$, en la dirección $x \mathrm{y}$ en el instante $t$, con la configuración referencial de longitud $L$. Supongamos que el material es de tipo viscoso por lo tanto la ley constitutiva que relaciona tensión y deformación es

$$
\theta=\alpha u_{x}+\gamma u_{x t} .
$$

de donde deducimos que su ecuación momento viene dado por,

$$
u_{t t}-\alpha u_{x x}-\gamma u_{x x t}=0, \quad(x, t) \in(0, L) \times(0, \infty) .
$$

con $\alpha, \gamma$ constantes reales positivas.

Si la barra se sujeta en ambos extremos, $x=0$ y $x=L$, entonces las condiciones de frontera son expresadas por,

$$
u(0, t)=0, u(L, t)=0,
$$

Observemos que si en vez de $\gamma$, pusiéramos $-a(x)$, con $a \in L_{+}^{\infty}(0, L)$ y $a(x) \geq a_{0}>0$, en la ecuación anterior, entonces el amortiguamiento es distribuido sobre toda la barra.

En la práctica, es suficiente considerar el amortiguamiento localmente distribuido, ver [7]. Nosotros trabajamos considerando $\gamma=\gamma(x) \in W^{2, \infty}$.

Por lo que plantearemos el problema viscoelástica con condiciones iniciales y de frontera siguiente:

$$
\mid \begin{aligned}
& u_{t t}-\alpha u_{x x}-\gamma(x) u_{x x t}=0, \quad(x, t) \in(0, L) \times(0, \infty) \\
& u(0, t)=u(L, t)=0, \quad t>0 \\
& u(x, 0)=u_{0}(x), \quad \text { en }(0, L) \\
& u_{t}(x, 0)=u_{1}(x), \quad \text { en }(0, L)
\end{aligned}
$$

\footnotetext{
${ }^{1}$ UNMSM, Facultad de Ciencias Matemáticas, e-mail: apersal@hotmail.com

${ }^{2}$ UNMSM, Facultad de Ciencias Matemáticas, e-mail: victoriano_yauri@hotmail.com

${ }^{3}$ UNMSM, Facultad de Ciencias Matemáticas, e-mail: e-mail: agcbayo@yahoo.es

${ }^{4}$ UNMSM, Facultad de Ciencias Matemáticas, e-mail: ebrunmsm@hotmail.com
} 
donde $\gamma \in W^{2, \infty}$ cumple con las siguientes condiciones:

C1) Existe $\gamma_{0} \in \mathbb{R}$ tal que $\gamma(x) \geq \gamma_{0}>0$ en $[0, L]$,

C2) $\gamma^{\prime \prime}(x) \leq 0$ en $[0, L]$.

El estudio con disipación de tipo $\gamma$ como constante, fue realizado por Zheng-Liu en 1999, ver [17] y $\gamma(x)$ como una función lineal, fue estudiado por Cunha-Diniz, ver [3]. Ellos demostraron la estabilidad exponencial del semigrupo asociado a una ecuación lineal viscoelástica. Nosotros aportaremos, mejorando el estudio realizado por Zheng-Liu, con $\gamma=\gamma(x) \in W^{2, \infty}$.

\section{Notaciones y Resultados Previos}

Sea $A$ un operador definido sobre un espacio de Banach $X$. El conjunto resolvente de $A$ es el conjunto de los $\lambda \in \mathbb{C}$, para los cuales el operador lineal $\lambda I-A$ es inversible, con inverso acotado y tiene dominio denso en $X$, se denota por $\rho(A)$.

Damos algunos teoremas que serán usados en la siguientes secciones

Teorema 1 (Hille -Yosida) Un operador lineal A, no acotado es un generador infinitesimal de un semigrupo $C_{0}$ de contracciones si, y sólo si,

i) $A$ es cerrado y $\overline{D(A)}=X$.

ii) El conjunto resolvente $\rho(A)$ de $A$ contiene al conjunto $\mathbb{R}^{+}$y para todo $\lambda>0$, es válido

$$
\left\|(\lambda I-A)^{-1}\right\| \leq \frac{1}{\lambda}
$$

Demostración. Ver [9], pág. 63

Teorema 2 Sea $A$ un operador lineal (no acotado), disipativo y con dominio denso en $X$. Si $0 \in \rho(A)$, entonces $A$ es el generador infinitesimal de un semigrupo $C_{0}$ de contracciones.

Demostración. Ver [9], pág. 88

Sea $A$ un operador lineal de $D(A) \subset E$ en $E$. Dado $x_{0} \in E$ el problema de Cauchy para $A$ con valor inicial $x_{0}$ consiste en encontrar una solución $u(t)$ para el problema de valor inicial

$$
\mid \begin{gathered}
\frac{d u(t)}{d t}=A u(t), \quad t>0 \\
u(0)=x_{0}
\end{gathered}
$$

Notar que como $u(t) \in D(A)$ para $t>0$ y $u$ es continua en $t=0$, el sistema no puede tener una solución para $x \notin \overline{D(A)}$.

Teorema 3 Sea $S(t)$ un $C_{0}$ semigrupo y sea $A$ su generador infinitesimal . Entonces

a) Para $x \in E$,

$$
\lim _{h \rightarrow 0} \frac{1}{h} \int_{t}^{t+h} S(s) x d s=S(t) x .
$$

b) Para $x \in E, \int_{0}^{t} S(s) x d s \in D(A) y$

$$
A\left(\int_{0}^{t} S(s) x d s\right)=S(t) x-x .
$$

c) Para $x \in D(A), S(t) x \in D(A) y$

$$
\frac{d}{d t} S(t) x=A S(t) x=S(t) A x .
$$


d) Para $x \in D(A)$,

$$
S(t) x-S(s) x=\int_{s}^{t} S(\tau) A x d \tau=\int_{s}^{t} A S(\tau) x d \tau
$$

Demostración. Ver [14], pág. 44

Por el teorema anterior tenemos que el operador $A$, es el generador infinitesimal de un $C_{0}$-semigrupo $S$, el problema de Cauchy para $A$ tiene una solución denotada por $u(t, x)=S(t) x$ para todo $x \in D(A)$. Ahora para mostrar que $x \in D(A), u(t, x)=S(t) x$ es la única solución del problema de valor inicial dado en (2), se usará el siguiente teorema.

Teorema 4 Si A es el generador infinitesimal de un semigrupo diferenciable para todo $x \in E$ el problema de valor inicial (2) tiene una única solución, cuando $x_{0} \in D(A)$.

Demostración. Ver [14], pág. 104

Teorema 5 Sean $A$ el generador infinitesimal de un semigrupo diferenciable y $S(t)=e^{A t}$ un semigrupo $C_{0}$ de contracciones definido en un espacio de Hilbert. Entonces $S(t)$ es exponencialmente estable si, y sólo si,

$$
\begin{aligned}
\rho(A) & \supseteq\{i \beta: \beta \in \mathbb{R}\} \equiv i \mathbb{R} \\
\overline{\lim }_{|\beta| \rightarrow \infty}\left\|(i \beta I-A)^{-1}\right\| & <\infty
\end{aligned}
$$

Demostración. Ver [17], pág.4

\section{Existencia, Unicidad y Estabilidad Exponencial}

Para transformar el problema (1) en una ecuación autónoma introduciremos la notación $v=u_{t}$, $\Delta u=u_{x x}$ y consideraremos el espacio de Hilbert

$$
H=H_{0}^{1}(0, L) \times L^{2}(0, L)
$$

con $H_{0}^{1}(0, L)$ provisto de la norma $\|u\|_{H_{0}^{1}}^{2}=\int_{0}^{L} \alpha\left|u_{x}\right|^{2} d x$ y $H$ provisto del producto interno

$$
\left(U_{1}, U_{2}\right)_{H}=\int_{0}^{L} \alpha u_{x} \cdot y_{x} d x+\int_{0}^{L} v \cdot z d x
$$

con $U_{1}=(u, v)^{T}, U_{2}=(y, z)^{T} \in H$.

El problema (1) puede ser reducido a un problema de Cauchy con condición inicial en el espacio de Hilbert $H$. Así obtenemos un operador autónomo para poder aplicar la teoría de semigrupos al P.V.I. siguiente

$$
\mid \begin{aligned}
& \frac{d U}{d t}=A U, \quad t>0 \\
& \left.U\right|_{t=0}=U_{0}
\end{aligned}
$$

donde

$$
\begin{gathered}
A=\left(\begin{array}{cc}
0 & I \\
\alpha \Delta & \gamma(x) \Delta
\end{array}\right), U=(u, v)^{T} \\
\left.U\right|_{t=0}=\left(u_{0}, u_{1}\right)^{T} .
\end{gathered}
$$

con

$$
D(A)=\left\{y=(u, v)^{T} \in H / v \in H_{0}^{1}(0, L), \alpha u_{x}+\gamma(x) v_{x} \in H^{1}(0, L)\right\} .
$$

Proposición 1 El operador A es disipativo. 
Demostración. Sea $U=(u, v)^{T} \in D(A)$.

$$
(A U, U)_{H}=\int_{0}^{L} \alpha v_{x} u_{x} d x+\int_{0}^{L}\left(\alpha u_{x x}+\gamma(x) v_{x x}\right) v d x .
$$

Haciendo integración por partes para $\alpha u_{x}+\gamma(x) v_{x}$ y $v \in H^{1}(0, L)$, tenemos

$$
\begin{gathered}
\int_{0}^{L}\left(\alpha u_{x}+\gamma(x) v_{x}\right)_{x} v d x=-\int_{0}^{L}\left(\alpha u_{x}+\gamma(x) v_{x}\right) v_{x} d x \\
(A U, U)_{H}=-\int_{0}^{L} \gamma(x) v_{x}^{2} d x+\frac{1}{2} \int_{0}^{L} \gamma^{\prime \prime}(x) v^{2} d x
\end{gathered}
$$

y usando $(C 1)$ y $(C 2)$ en el lado derecho de (8) tenemos

$$
(A U, U)_{H} \leq 0 .
$$

Teorema 6 El operador $A$ definido en (6) es un generador infinitesimal de un $C_{0}$ - semigrupo de contracción en $H$.

Demostración. Como $H_{0}^{1}(0, L) \cap H^{2}(0, L)$ es denso en $H_{0}^{1}(0, L)$ y $L^{2}(0, L)$ con sus correspondientes normas y

$$
\left(H_{0}^{1}(0, L) \cap H^{2}(0, L)\right) \times\left(H_{0}^{1}(0, L) \cap H^{2}(0, L)\right) \subset D(A) \subset H,
$$

entonces

$$
D(A) \text { es denso en } H \text {. }
$$

Luego, sea $F=(f, g)^{T} \in H$, consideremos la ecuación $A U=F$, es decir,

$$
\begin{aligned}
v & =f \in H_{0}^{1}(0, L) \\
\alpha u_{x x}+\gamma(x) v_{x x} & =g \in L^{2}(0, L) \hookrightarrow H^{-1}(0, L) .
\end{aligned}
$$

Tomamos $v=f$, obtenemos

$$
\alpha u_{x x}+\gamma(x) f_{x x}=g \in H^{-1}(0, L) .
$$

Evaluando

$$
\int_{0}^{L}\left(\alpha u_{x x}+\gamma(x) f_{x x}\right) w d x=\int_{0}^{L} g w d x, \forall w \in H_{0}^{1}(0, L) .
$$

Efectuando integración por partes

$$
\begin{gathered}
\int_{0}^{L}\left(\alpha u_{x}+\gamma(x) f_{x}\right)_{x} w d x=-\int_{0}^{L}\left(\alpha u_{x}+\gamma(x) f_{x}\right) w_{x} d x \\
\int_{0}^{L} \underbrace{\alpha u_{x x}+\gamma(x) f_{x x}}_{g}+\gamma^{\prime}(x) f_{x}) w d x=-\int_{0}^{L}\left(\alpha u_{x}+\gamma(x) f_{x}\right) w_{x} d x .
\end{gathered}
$$

Sea $G=g+a f_{x} \in L^{2}(0, L), F=\gamma(x) f_{x} \in L^{2}(0, L)$ y reemplazando en (9) tenemos

$$
\int_{0}^{L} \alpha u_{x} w_{x} d x=-\int_{0}^{L} G w d x-\int_{0}^{L} F w_{x} d x ; \forall w \in H_{0}^{1}(0, L) .
$$

Definimos

y

$$
\begin{array}{ccc}
a(., .): \quad H_{0}^{1}(0, L) \times H_{0}^{1}(0, L) & \rightarrow \quad \mathbb{R} \\
(u, w) & \mapsto a(u, w)=\alpha \int_{0}^{L} u_{x} w_{x} d x
\end{array}
$$

$$
\begin{array}{clc}
\varphi: H_{0}^{1}(0, L) & \rightarrow & \mathbb{R} \\
w & \mapsto\langle\varphi, w\rangle_{H^{-1} \times H_{0}^{1}}= & -\int_{0}^{L} G w d x-\int_{0}^{L} F w_{x} d x
\end{array}
$$


Así en (10) tenemos

$$
a(u, v)=\langle\varphi, w\rangle \quad ; \quad \forall w \in H_{0}^{1}(0, L) .
$$

Por el teorema de Lax-Milgran tenemos que existe un único $u \in H_{0}^{1}(0, L)$ tal que

$$
a(u, w)=\langle\varphi, w\rangle \quad \forall w \in H_{0}^{1}(0, L) .
$$

Entonces existe un único $(u, v)^{T} \in H_{0}^{1}(0, L)$ tal que

$$
\alpha u_{x x}+\gamma(x) v_{x x}=g \wedge v=f .
$$

Entonces $A$ es inversible.

Sea $U=(u, v)^{T} \in D(A)$

$$
\begin{gathered}
\|U\|_{H}^{2}=\|u\|_{H_{0}^{1}}^{2}+\|v\|_{L^{2}}^{2} \\
\|U\|_{H}^{2}=\frac{1}{\alpha}\left\|\alpha u_{x}\right\|_{L^{2}}^{2}+\|v\|_{L^{2}}^{2} \\
\|U\|_{H}^{2}=\frac{1}{\alpha}\left\|\alpha u_{x}+\gamma(x) v_{x}-\gamma(x) v_{x}\right\|_{L^{2}}^{2}+\|v\|_{L^{2}}^{2} \\
\|U\|_{H}^{2} \leq \frac{1}{\alpha}\left(\left\|\alpha u_{x}+\gamma(x) v_{x}\right\|_{L^{2}}+\left\|\gamma(x) v_{x}\right\|_{L^{2}}\right)^{2}+\|v\|_{L^{2}}^{2} .
\end{gathered}
$$

Desde que $(x+y)^{2} \leq 2\left(x^{2}+y^{2}\right)$ y $\left\|\gamma(x) v_{x}\right\|_{L^{2}}^{2} \leq\|\gamma\|_{L^{\infty}}^{2}\left\|v_{x}\right\|_{L^{2}}^{2}$, se tiene

$$
\|U\|_{H}^{2} \leq \frac{2}{\alpha}\left\|\alpha u_{x}+\gamma(x) v_{x}\right\|_{L^{2}}^{2}+\frac{2}{\alpha}\|\gamma\|_{L^{\infty}}^{2}\left\|v_{x}\right\|_{L^{2}}^{2}+\|v\|_{L^{2}}^{2} .
$$

Aplicando la desigualdad de Poincaré y $(x+y)^{2} \leq 2\left(x^{2}+y^{2}\right)$ tenemos

$$
\|U\|_{H}^{2} \leq \frac{4 K^{2}}{\alpha^{2}}\left\|\alpha u_{x x}+\gamma(x) v_{x x}\right\|_{L^{2}}^{2}+\left(\frac{4 K^{2}}{\alpha^{2}}\left\|\gamma^{\prime}\right\|_{L^{\infty}}^{2}+\frac{2}{\alpha}\|\gamma\|_{L^{\infty}}^{2}+\frac{1}{\alpha} K^{2}\right)\left\|v_{x}\right\|_{L^{2}}^{2} .
$$

Tomamos $M^{2}=\operatorname{máx}\left\{\frac{9 K^{2}}{\alpha^{2}}, \frac{4 K^{2}}{\alpha^{2}}\left\|\gamma^{\prime}\right\|_{L^{\infty}}^{2}+\frac{2}{\alpha}\|\gamma\|_{L^{\infty}}^{2}+\frac{1}{\alpha} K^{2}\right\}$ tenemos

$$
\|U\|_{H}^{2} \leq M^{2}\left(\left\|\alpha u_{x x}+\gamma(x) v_{x x}\right\|_{L^{2}}^{2}+\left\|v_{k}\right\|_{H_{0}^{1}}^{2}\right)
$$

es decir,

$$
\|U\|_{H}^{2} \leq M^{2}\|A U\|_{H}^{2} ; \forall U \in D(A)
$$

y como $A$ es inversible tenemos

$$
\left\|A^{-1} F\right\|_{H} \leq M\|F\|_{H} ; \forall F \in H
$$

Entonces $A^{-1}$ es acotado.

Como $A$ es inversible y $A^{-1}$ es acotado, entonces

$$
0 \in \rho(A) .
$$

Como $A^{-1}$ es inversible y acotado entonces $A$ es acotado.

\section{En resumen:}

Hemos verificado que $D(A)$ es denso en $H, 0 \in \rho(A)$ y por la proposición $1, A$ es disipativo; luego, por el teorema 3 , concluimos que el operador $A$ es un generador infinitesimal de un $C_{0}$ - semigrupo $S(t)$ de contracciones en $H$, que se denota $S(t)$ por $e^{A t}, t \geq 0$.

Como $A$ es un operador cerrado, entonces $\left(D(A),\|\cdot\|_{D(A)}\right)$ es un Espacio de Banach con la norma del gráfico, esto es ,

$$
\|w\|_{D(A)}^{2}=\|w\|_{H}^{2}+\|A w\|_{H}^{2} .
$$

Si tomamos $w=U_{0} \in D(A)$ y usando el teorema 5 tenemos

$$
\exists ! U \in C^{1}(0, \infty, H) \cap C(0, \infty, D(A))
$$

lo cual resuelve el problema de Cauchy (5). Con los resultados obtenidos veremos la estabilidad exponencial del $C_{0}$ - semigrupo de contracción $(S(t))_{t>0}$ generado por $A$, asociado a la ecuación viscoelástica lineal con amortiguamiento localmente distribuido. 


\section{Proposición 2}

$$
\left\{i \beta \in i \mathbb{R} /|\beta|<\left\|A^{-1}\right\|^{-1}\right\} \subset \rho(A) .
$$

Demostración. Sea $\beta \in \mathbb{R}$ tal que $|\beta|<\left\|A^{-1}\right\|^{-1}$, entonces

$$
\left\|i \beta A^{-1}\right\|<1 .
$$

De (11) tenemos $i \beta A^{-1}-I$ es inversible y continua . Como $A$ es inversible y continua entonces

$$
i \beta I-A=\left(i \beta A^{-1}-I\right) A
$$

es inversible y continua, luego

$$
i \beta \in \rho(A) \text {. }
$$

Proposición 3 Sea $w \in \mathbb{R}^{+}$. Si

$$
\{i \beta \in i \mathbb{R} /|\beta|<w\} \subset \rho(A)
$$

$y$

$$
\sup \left\{\left\|(i \beta I-A)^{-1}\right\| /|\beta|<w\right\}=M<\infty,
$$

entonces

$$
i w \in \rho(A) .
$$

Demostración. Sea $\beta_{0} \in \mathbb{R}$ tal que $\left|\beta_{0}\right|<w$, entonces

$$
i \beta_{0} \in \rho(A) \text {. }
$$

Si $\left\|i\left(\beta-\beta_{0}\right)\left(i \beta_{0} I-A\right)^{-1}\right\|<1$, entonces

$$
I+i\left(\beta-\beta_{0}\right)\left(i \beta_{0} I-A\right)^{-1} \text { es inversible y continua. }
$$

Por (12), (13) y como

$$
i \beta I-A=\left(i \beta_{0} I-A\right)\left(I+i\left(\beta-\beta_{0}\right)(i \beta I-A)^{-1}\right)
$$

entonces

$$
i \beta I-A \text { es inversible. }
$$

Tenemos que $\left\|\left(i \beta_{0} I-A\right)^{-1}\right\| \leq M$ y usando (14) obtenemos

si $\left|\beta_{0}\right|<w$ y $\left|\beta-\beta_{0}\right|<M^{-1}$ se tiene $i \beta I-A$ es inversible.

Tomemos $\beta_{0}=w-\epsilon$ donde $\epsilon=\frac{1}{2} \operatorname{mín}\left\{w, M^{-1}\right\}$, entonces

$$
0<\beta_{0}<w \wedge\left|w-\beta_{0}\right|<M^{-1}
$$

y aplicando (15), se obtiene

$$
i w \in \rho(A) .
$$

Teorema 7 El $C_{0}$ - semigrupo de contracciones $(S(t))_{t>0}$ generado por A es exponencialmente estable; es decir, existen constantes positivas $M$ y w tal que $\|S(t)\| \leq M e^{-w t}$.

Demostración. Aplicaremos el teorema 5, a fin de verificar que:

a) $\rho(A) \supseteq\{i \beta: \beta \in \mathbb{R}\} \equiv i \mathbb{R}$

b) $\overline{\lim }_{|\beta| \rightarrow \infty}\left\|(i \beta I-A)^{-1}\right\|<\infty$ 
a) Demostraremos por reducción al absurdo.

Supongamos que $i \mathbb{R} \nsubseteq \rho(A)$, entonces existe $\beta^{\prime} \in \mathbb{R}$ tal que

$$
i \beta^{\prime} \notin \rho(A) \text {. }
$$

Sea

$$
C=\{|\beta| / \beta \in \mathbb{R} \wedge i \beta \notin \rho(A)\}
$$

donde $C \neq \phi$, pues $\left|\beta^{\prime}\right| \in C$.

Sea $|\beta| \in C$, entonces

$$
i \beta \notin \rho(A) .
$$

Tenemos dos opciones

$$
|\beta|<\left\|A^{-1}\right\|^{-1} \vee|\beta| \geq\left\|A^{-1}\right\|^{-1} .
$$

Si $|\beta|<\left\|A^{-1}\right\|^{-1}$ y por la proposición 3 tendríamos que $i \beta \in \rho(A)$ pero eso no puede suceder por (16). Si $|\beta| \geq\left\|A^{-1}\right\|^{-1}$, entonces el conjunto $C$ está acotado inferiormente, entonces existe un ínfimo del conjunto $C$ y lo denotamos por $w$, aún más $w \in C$.

Como $w=\operatorname{mín} C$, entonces

$$
\{i \beta \in i \mathbb{R} /|\beta|<w\} \subset \rho(A) .
$$

Sea

$$
D=\left\{\left\|(i \beta I-A)^{-1}\right\| /|\beta|<w\right\} .
$$

Por la proposición 2 tenemos que

$$
\sup _{\substack{\beta \in \mathbb{R} \\|\beta|<w}} D=\infty
$$

pues caso contrario tendríamos que $i w \in \rho(A)$ de la proposición 3; pero $w \in C$, es clecir, $i w \notin \rho(A)$. Tomemos $\left\{\beta_{n}\right\}_{n \in \mathbb{N}} \subset \mathbb{R}$ tal que

$$
\beta_{n} \rightarrow w \wedge\left|\beta_{n}\right|<w .
$$

Como $\left|\beta_{n}\right|<w$ y por (14) tenemos

$$
\left\{i \beta_{n}\right\} \subset \rho(A) .
$$

Entonces con esta sucesión $\left\{\beta_{n}\right\}_{n \in \mathbb{N}}$ podemos encontrar una sucesión

$$
\left\{U_{n}\right\}_{n \in \mathbb{N}}=\left\{\left(u_{n}, v_{n}\right)^{T}\right\}_{n \in \mathbb{N}} \subset D(A)
$$

tal que

$$
\left\|U_{n}\right\|=1 \wedge\left\|\left(i \beta_{n} I-A\right) U_{n}\right\|_{H} \rightarrow 0 \text { cuando } n \rightarrow \infty .
$$

Por (18) tenemos

$$
\begin{aligned}
i \beta_{n} u_{n}-v_{n} & \rightarrow 0 \text { en } H_{0}^{1}(0, L) \\
i \beta_{n} v_{n}-\alpha u_{n x x}-\gamma(x) v_{n x x} & \rightarrow 0 \mathrm{en} L^{2}(0, L) .
\end{aligned}
$$

Por otro lado tomamos la parte real del producto interno de $\left(i \beta_{n} I-A\right) U_{n}$ con $U_{n}$ en $H$

$$
\begin{gathered}
\operatorname{Re}\left(\left(\left(i \beta_{n} I-A\right) U_{n}, U_{n}\right)_{H}\right)=-\operatorname{Re}\left(\left(A U_{n}, U_{n}\right)_{H}\right) \\
\operatorname{Re}\left(\left(\left(i \beta_{n} I-A\right) U_{n}, U_{n}\right)_{H}\right)=\int_{0}^{L} \gamma(x) v_{n x}^{2} d x-\frac{1}{2} \int_{0}^{L} \gamma^{\prime \prime}(x) v_{n}^{2} d x .
\end{gathered}
$$

Como $\left(i \beta_{n} I-A\right) U_{n} \rightarrow 0$ en $H$ y $\left\|U_{n}\right\|_{H}=1$ entonces

$$
\int_{0}^{L} \gamma(x) v_{n x}^{2} d x-\frac{1}{2} \int_{0}^{L} \gamma^{\prime \prime}(x) v_{n}^{2} d x \rightarrow 0
$$


Por (C1) y (C2) tenemos

$$
0<\int_{0}^{L} \gamma_{0} v_{n x}^{2} \leq \int_{0}^{L} \gamma(x) v_{n x}^{2} d x-\frac{1}{2} \int_{0}^{L} \gamma^{\prime \prime}(x) v_{n}^{2} d x .
$$

De (21) y (22) tenemos

$$
v_{n} \rightarrow 0 \text { en } H_{0}^{1}(0, L) .
$$

De (18) y (23) tenemos

$$
\beta_{n} u_{n} \rightarrow 0 \text { en } H_{0}^{1}(0, L)
$$

y como $\beta_{n} \rightarrow w$, entonces

$$
u_{n} \rightarrow 0 \text { en } H_{0}^{1}(0, L)
$$

De (23) y (24) tenemos

$$
\left\|U_{n}\right\|_{H}^{2}=\left\|u_{n}\right\|_{H_{0}^{1}}^{2}+\left\|v_{n}\right\|_{L^{2}}^{2} \rightarrow 0
$$

pero $\left\|U_{n}\right\|_{H}^{2}=1$ entonces llegamos a una contradicción.

Por lo tanto

$$
\rho(A) \supseteq\{i \beta / \beta \in \mathbb{R}\} .
$$

b) Demostraremos por reducción al absurdo.

Supongamos que,

$$
\lim _{|\beta| \rightarrow \infty} \sup \left\|(i \beta I-A)^{-1}\right\|=\infty .
$$

Tomemos $\left\{\beta_{n}\right\}_{n \in \mathbb{N}} \subset \mathbb{R}$ tal que $\left|\beta_{n}\right| \rightarrow \infty$ y por (25) tenemos una sucesión

$$
\left\{U_{n}\right\}_{n \in \mathbb{N}} \subset D(A)
$$

tal que

$$
\left\|U_{n}\right\|=1 \wedge\left\|\left(i \beta_{n} I-A\right)^{-1} U_{n}\right\|_{H} \rightarrow 0 .
$$

Tomamos la parte real del producto interno de $\left(i \beta_{n} I-A\right) U_{n}$ con $U_{n}$ en $H$ y obtenemos

$$
\operatorname{Re}\left(\left(\left(i \beta_{n} I-A\right) U_{n}, U_{n}\right)_{H}\right)=\int_{0}^{L} \gamma(x) v_{n x}^{2} d x-\frac{1}{2} \int_{0}^{L} \gamma^{\prime \prime}(x) v_{n}^{2} d x .
$$

Como $\left(i \beta_{n} I-A\right) U_{n} \rightarrow 0$ en $H$ y $\left\|U_{n}\right\|_{H}=1$, entonces

$$
\int_{0}^{L} \gamma(x) v_{n x}^{2} d x-\frac{1}{2} \int_{0}^{L} \gamma^{\prime \prime}(x) v_{n}^{2} d x \rightarrow 0
$$

Por $(\mathrm{C} 1)$ y $(\mathrm{C} 2)$ tenemos

$$
0<\int_{0}^{L} \gamma_{0} v_{n x}^{2} \leq \int_{0}^{L} \gamma(x) v_{n x}^{2} d x-\frac{1}{2} \int_{0}^{L} \gamma^{\prime \prime}(x) v_{n}^{2} d x .
$$

De (27) y (28) tenemos

$$
v_{n} \rightarrow 0 \text { en } H_{0}^{1}(0, L)
$$

y como $\beta_{n} \rightarrow \infty$, entonces

$$
u_{n} \rightarrow 0 \text { en } H_{0}^{1}(0, L)
$$

De (29) y (30) tenemos

$$
U_{n} \rightarrow 0 \text { en } H
$$

pero $\left\|U_{n}\right\|_{H}^{2}=1$ entonces llegamos a una contradicción.

Por lo tanto

$$
\lim _{|\beta| \rightarrow \infty} \sup \left\|(i \beta I-A)^{-1}\right\|<\infty
$$

Así hemos verificado (a) y (b) que son las condiciones para usar el teorema 5 entonces el $C_{0}$ - semigrupo de contracción $(S(t))_{t>0}$ generado por $A$ es exponencialmente estable, con lo cual queda demostrado el teorema 7. 


\section{Conclusiones}

1. Se ha demostrado que al trabajo realizado por Zheng-Liu en 1999, cambiando la constante real positiva $\gamma$ en el término disipativo $u_{x x t}$ por una función que depende de la variable espacial " $x$ ", esto es, considerar $\gamma(x) u_{x x t}$, como disipación interna; también se puede obtener el mismo resultado obtenido por Zheng-Liu, con la única condición de que $\gamma(x) \in W^{2, \infty}$.

2. Se puede observar en el trabajo que, para el estudio de la existencia, unicidad y la estabilidad exponencial del sistema planteado podemos trabajar solamente con la teoría de semigrupos lineales. 


\section{REFERENCIAS BIBLIOGRÁFICAS}

[1] ADAMS, R. A. (1975). Sobolev Spaces. Academic Press, New York.

[2] BREZIS, H. (1984). Análisis Funcional, Teoría y Aplicaciones. Alianza Editorial, S.A. Madrid.

[3] CARLOS R. ; ADELIA D. (2006). Exponential Stability for Semigroup Associated with a Linear Viscoelastic Equation by a Locally Distributed Damping. Rev. Mat.Estal., Sao Paulo.

[4] DUNFORD, N.; SCHWARTZ, J. T. (1958). Linear Operators. Interscience Publishers Inc., New York.

[5] DAUTRAY, R.; LIONS, J. (1988). Mathematical Analysis and Numerical Methode for Science and Technology. Springer - Verlag Berlin Heiderlberg.

[6] EBERHARD Z. (1990). Nonlinear Functional Analysis and its Applications. Springer Verlag New York Inc.

[7] ENRIQUE Z. (1990). Exponential decay for the semilinear wave equation with locally distributed damping. Commun. PDE, London.

[8] JAIME MUÑOZ. (2004). Teoria das Distribuições e Equações Diferenciais Parciais. Textos Avanzados-LNCC. Petrópolis-Rio de Janeiro.

[9] JAIME MUÑOZ. (2008). Estabilização de Semigrupos e Aplicações. Edit. Acaclemia das Contas, Rio de Janeiro.

[10] LIU, K.; RAO B.; ZHANG, X. (2002). Stabilization of the wave equations with potential and indefinite damping. J. Math. Anal. Appl.

[11] LUIZ A. MEDEIROS. (1968). Tópicos de Análise Funcional. Instituto de Matemática, Univ. Federal de Pernambuco.

[12] LUIZ A. MEDEIROS (1989). A Integral de Lebesgue. Universidade Federal do Rio de Janeiro, Centro de Ciências Matemáticas e da Natureza, Instituto de Matemática.

[13] MORRIS, W. H.; STEPHENS, S. (1974). Differential Equations, Dynamical Systems, and Linear Algebra. Academic Press, Inc.

[14] PAZY, A. (1983). Semigroups of Linear Operators and Applications to Partial Differential Equations. Applied Mathematical Sciences 44. Springer.

[15] WALTER R. (1973). Functional Analysis. McGraw-Hill, Inc.

[16] YOSIDA, K. (1980). Functional Analysis. 6th Ed., Springer-Verlag.

[17] ZHENG, S.; LIU, Z. (1999). Semigroups associated with dissipative systems. Chapman \& Hall CRC Research Notes in Mathematics. 398. Boca Raton. Fl. 